NIST Technical Note 2058

\title{
IREX Validation Dataset 2019
}

\author{
James R. Matey \\ George W. Quinn \\ Patrick J. Grother
}

This publication is available free of charge from:

https://doi.org/10.6028/NIST.TN.2058

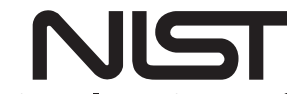

National Institute of Standards and Technology

U.S. Department of Commerce 
NIST Technical Note 2058

\title{
IREX Validation Dataset 2019
}

\author{
James R. Matey \\ George W. Quinn \\ Patrick J. Grother \\ Information Access Division \\ Information Technology Laboratory
}

This publication is available free of charge from:

https://doi.org/10.6028/NIST.TN.2058

September 2019

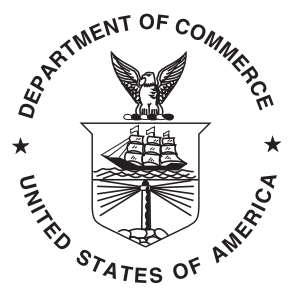

U.S. Department of Commerce Wilbur L. Ross, Jr., Secretary

National Institute of Standards and Technology Walter Copan, NIST Director and Undersecretary of Commerce for Standards and Technology 
Certain commercial entities, equipment, or materials may be identified in this document in order to describe an experimental procedure or concept adequately. Such identification is not intended to imply recommendation or endorsement by the National Institute of Standards and Technology, nor is it intended to imply that the entities, materials, or equipment are necessarily the best available for the purpose.

National Institute of Standards and Technology Technical Note 2058

Natl. Inst. Stand. Technol. Tech. Note 2058, 11 pages (September 2019)

CODEN: NTNOEF

This publication is available free of charge from: https://doi.org/10.6028/NIST.TN.2058 


\begin{abstract}
The IRis EXchange (IREX) program at the National Institute of Standards \& Technology (NIST) employs best measurement practices to support the development, standardization, and interoperability of iris-based technology through ongoing evaluations of that technology; IREX evaluations provide information that helps advance the development of a marketplace of iris-based applications based on standardized interoperable iris imagery and provides decision makers with information critical to their decisions about deployment of the technology.
\end{abstract}

IREX has conducted a series of evaluations of iris algorithms which are documented on the IREX website at https://www.nist.gov/programs-projects/iris-exchange-irex-overview.

To assure that algorithms submitted for evaluation produce the same results in the NIST evaluation environment that they do in the developer/vendor environment, we need to compare results on a common set of iris images in the two environments.

IREX-Validation-2019 is a dataset developed to facilitate such comparisons. In brief:

- The images in the dataset were collected under protocols approved by the NIST Institutional Review Board.

- The images are from subjects who provided model releases that permit use of the images in publications and datasets that are distributed by NIST.

- The dataset is distributed under user agreements associated with IREX evaluations.

- Inquiries regarding the IREX program may be directed to the IREX Liaison at irex@ nist.gov.

- Any papers or reports making use of the dataset should cite this report as discussed in section 2.2.

\title{
Key words
}

biometric, iris, recognition, evaluation, IREX, dataset. 


\section{Table of Contents}

1 Background 1

2 IREX Validation Dataset 2019

光. 2.2 Availability and Citation of Use 5

References $\quad 5$ 


\section{Glossary}

IRB Institutional Review Board. An Institutional Review Board conducts prospective and continuing review and approval of non-exempt research involving human subjects to ensure that the research is performed in accordance with applicable regulations and ethical principles; to protect the safety, rights, and welfare of participants; and, to prevent the unethical treatment of participants that might lead to various harms.

model release A model release is a legal release signed by the subject of a photograph that grants permission to publish the photograph in one form or the other. In this context a model release is part of an IRB approved consent form signed by the subject of a biometric data collection; the release grants permission from the subject for the use of their images in published datasets and published papers.

participant In this context, entities who submit algorithms for evaluation in the IREX evaluation program.

subject In this context, an individual who volunteered to provide iris images under a NIST IRB approved protocol. 


\section{Background}

The IRis EXchange (IREX) program at the National Institute of Standards \& Technology (NIST) employs best measurement practices to support the development, standardization, and interoperability of iris-based technology through ongoing evaluations of that technology; IREX evaluations provide information that helps advance the development of a marketplace of iris-based applications based on standardized interoperable iris imagery and provides decision makers with information critical to their decisions about deployment of the technology.

IREX has conducted a series of evaluations of iris algorithms which are documented on the IREX website at https://www.nist.gov/programs-projects/iris-exchange-irex-overview.

IREX makes use of three general categories of datasets in its iris evaluations:

- Validation datasets are small datasets distributed to evaluation participants; the participants run their to-be-submitted algorithms on the datasets in their environment and submit the results to NIST. NIST runs the participants' submitted algorithms on the same datasets in the NIST evaluation environment and compares results with results submitted by the participants to assure that the algorithms are generating the same results in the NIST evaluation environment that they did in the participants' environment.

- Public datasets are datasets that are generally available to the biometric community. NIST may make use of such datasets in its evaluations, but generally does not have authority to re-distribute those datasets. Where such datasets are used in evaluations, the report on the results of the evaluation will have information on where the datasets can be obtained. Interested parties should contact the source of the dataset to obtain copies. Some of the images, in some of the public datasets have associated model releases which permit publication of the image. NIST sometimes uses such images to illustrate reports and presentations, but, as noted, does not have authority for other redistribution.

- Sequestered datasets are large datasets that NIST maintains in a physically, logically and administratively secured laboratory that is dedicated to conducting evaluations of biometric algorithms; the laboratory is secured to assure the security and integrity of (1) the biometric samples used in the evaluations and (2) the algorithms submitted by the vendors/developers who participate in the evaluations. These datasets are tightly controlled and are not distributed or published to anyone (including developers) to ensure that algorithms tested with the sequestered datasets cannot be trained/overtrained on the datasets. 
The results of NIST evaluations are published; copies can be downloaded for individual IREX evaluations from the IREX homepage at https:/www.nist.gov/programs-projects/ iris-exchange-irex-overview or by conducting a search on the keyword IREX at the NIST publications website at https://www.nist.gov/publications/search.

\section{IREX Validation Dataset 2019}

\subsection{Description}

In brief, IREX-Validation-2019 consists of four sections: (1) approximately 80 images acquired from 3 subjects in a typical office environment with time intervals between images ranging from minutes to days; (2) approximately 480 images from the same subjects acquired as the ambient illumination was purposefully varied from a dimly lit to a brightly lit office environment; (3) various manipulations of the 80 images as described below; (4) several Creative Commons/Open Content images that have superficial resemblance to the human iris. These images were acquired under protocol ITL-16-0002, approved by the NIST Institutional Review Board (IRB). The images in this dataset are from subjects who signed an optional model release ${ }^{1}$ that provides permission for NIST to (a) incorporate the subject's iris images in datasets for distribution to participants in NIST evaluations and (b) use the images in NIST publications.

In more detail:

- The images were acquired using an LG- $4000^{2}$ dual-eye iris camera in an office/laboratory setting.

- The dataset contains left/right iris images collected from 3 subjects.

- The subject identifiers are coded. The subject identifiers for IREX Validation Dataset 2019 were recoded from the original coding of the collected images; the original codebook is not provided with the dataset and the recoded codebook does not exist.

- The data is time-stamped with the date of acquisition.

- As noted some of the images have been deliberately manipulated to stress algorithms, e.g. non-conventional image size, simulated specularity(s), and simulation of other issues described in IREX V, see https://www.nist.gov/itl/iad/image-group/ irex-v-homepage.

\footnotetext{
${ }^{1}$ See glossary.

${ }^{2}$ See https://www.irisid.com/; as already noted, use of any particular hardware or software should not be considered an endorsement of that hardware or software by NIST.
} 
- The data includes images in which the ambient illumination was purposefully varied to generate dilation changes.

- The image format is png, a standard format defined in ISO/IEC 15948:2004, see https://www.iso.org/standard/29581.html, except as noted for Misc, see below.

- The image filenames are of the form:

<coded subject ID>_<eye: L/R>_<acquisition date-time>_<treatment>.png

- The treatments, applied using IrfanView $4.37^{3}$ are:

- Orig: Original, no treatment, as captured images without manipulation and in a normal ambient environment, 640x480.

- Ambient-n.nnn: Original, no treatment, as captured images without manipulation, captured with varying ambient illumination. The ambient is reported on a zero to one scale corresponding to dimly to brightly lit.

- Border: Orig set with border that pads the images to $1280 \times 960$.

- Contrast: Orig set with contrast reduced.

- Crop: Orig set with images cropped to $320 \times 240$ around the iris.

- Double: Orig set with images resized to $1280 \times 960$.

- Half: Orig set with images resized to $320 \times 240$.

- HFlip: Orig set with images flipped horizontally.

- RGB: Orig set with images converted to 24 bit RGB.

- Shift: Border set with images cropped to to $640 \times 480$, with offsets to push iris texture to image corners.

- VFlip: Orig set with images flipped vertically.

- Misc:

* There are 4 color images of subject C; with Color as the treatment. One L and one $\mathrm{R}$ in png and jpg formats. These were taken with a DSLR camera and cropped/resized to $640 \times 480$.

* There are 2 images of subject $\mathrm{C}$ with eyes closed; with Closed as the treatment. One $\mathrm{L}$ and one $\mathrm{R}$ in png format. These were taken with an IrisID7000 .

* There are 2 images of subject $\mathrm{C}$, intentionally presenting to generate a poor image; with PoorPresentation as the treatment. One $\mathrm{L}$ and one $\mathrm{R}$ in png format. These were taken with an IrisID-7000.

${ }^{3}$ https://www.irfanview.com/; as already noted, use of any particular hardware or software should not be considered an endorsement of that hardware or software by NIST. 
* There is one $1 \times 1$ pixel image generated using IrfanView, dummy subject $\mathrm{D}$, treatment 1pixel.

* There are three $640 \times 480$ Blank images generated using IrfanView - black, medium gray and white, dummy subject $\mathrm{D}$, treatment Blank-xxxx.

* Painting: "Irises“, by Vincent Van Gogh, from the J. Paul Getty Museum, http://media.getty.edu/museum/images/web/enlarge/00094701.jpg, distributed under Getty's Open Content Program, http://www.getty.edu/about/whatwedo/ opencontent.html; dummy subject $\mathrm{D}$, treatment Painting.

* Picture: From Creations by Kara, https://www.creationsbykara.com/wp-content/ uploads/2017/02/Funfetti-Donuts-004-1-625x788.jpg, modified slightly, distributed under Creative Commons: https://creativecommons.org/licenses/ by-nc-nd/3.0/us/legalcode; dummy subject D, treatment Picture.

* Drawing: From Allie Darum, https://commons.wikimedia.org/wiki/File: Eye_liner.jpg, modified slightly, distributed under Creative Commons: https: //creativecommons.org/licenses/by-sa/3.0/legalcode; dummy subject D, treatment Drawing.

This dataset is not intended to be representative of the datasets used in the IREX evaluations. It is not intended for training algorithms. It's purpose is to validate/verify that algorithms produce the same results in the NIST evaluation environment as in the vendor/developer environment - including proper handling of error conditions brought about by variation/abnormalities in the images provided to the algorithms.

In typical use, template generation would be attempted for all the images and a report generated that provides a success/failure code and diagnostics (e.g. pupil/iris locations and radii, template quality, image quality) for each image. All the resulting templates would then be compared to all the resulting templates and the results reported in a match score table with the original filenames for each image pair, the match score and the match quality. Details may vary with the particular evaluation - see the instructions for the particular evaluation for details. 


\subsection{Availability and Citation of Use}

The dataset will be provided, free of charge, to participants in IREX evaluations as part of the user agreement for the evaluation. The use of the dataset will be subject to the terms of the user agreement. See the IREX home page for announcements of evaluations and for access to the IREX email distribution list.

In any publication that reports results based on this dataset, please cite this paper as shown in the references[1]:

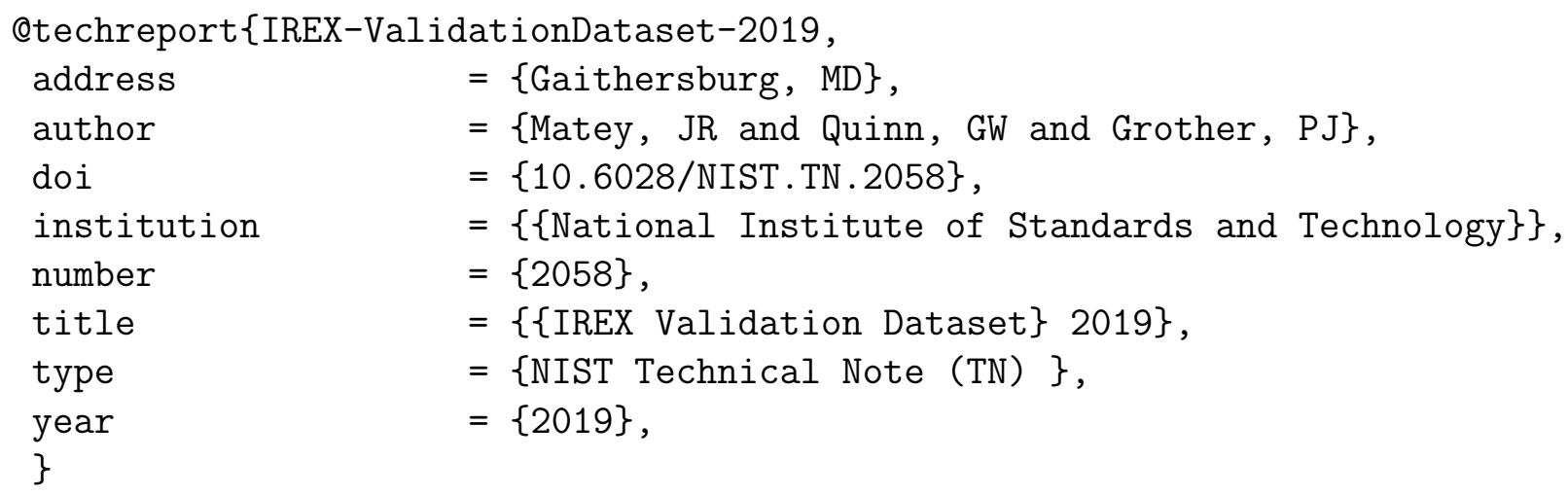

\section{Acknowledgments}

Our thanks to the subjects who participated in the collection of data under the NIST IRB approved protocol ITL-16-0002, especially those who agreed to the publication and distribution of their images.

Our thanks to John Howard of SAIC (Science Applications International Corporation) and to Mei Ngan of NIST for helpful comments on the manuscript.

\section{References}

[1] Matey J, Quinn G, Grother P (2019) IREX Validation Dataset 2019. National Institute of Standards and Technology Gaithersburg, MD, NIST Technical Note (TN) 2058. https://doi.org/10.6028/NIST.TN.2058 